\title{
Long-term remission with surgery for recurrent localized Hodgkin lymphoma
}

\author{
A. Kentos, MD, ${ }^{a}$ P. Rocmans, MD, PhD, ${ }^{b}$ M. Remmelink, $\mathrm{MD}, \mathrm{PhD}^{\mathrm{c}}$ and $\mathrm{W}$. Feremans, $\mathrm{MD}, \mathrm{PhD},{ }^{a}$ Brussels, Belgium
}

$\mathrm{P}$ atients with advanced Hodgkin disease (HD) who have a relapse after first-line chemotherapy can achieve further remissions with high-dose chemotherapy and transplantation of hematopoietic stem cells. Unfortunately, relapse or disease progression after autologous transplant occurs in a significant proportion of cases, and no clear treatment guidelines exist for these patients. We report the case of a patient with a refractory localized HD who had a sustained long-term remission (39 months) after surgery alone.

\section{Clinical Summary}

A 22-year-old male student was seen in November 1995 with B signs and superior vena cava syndrome. HD of the nodular sclerosis type was diagnosed at stage IIIB with bulky mediastinum. The patient was treated with 6 cycles of doxorubicin, bleomycin, vinblastine, and dacarbazine to achieve a complete remission, consolidated by 40-Gy radiotherapy to initial sites of bulky mediastinum and by $30-G y$ radiotherapy to neck and axillary areas. Two years after completion of therapy, the disease recurred as a localized left lung suprahilar nodule within the previously irradiated field. The patient was successfully treated with 3 courses of etoposide, vinblastine, cytarabine, and cisplatin chemotherapy, followed by high-dose chemotherapy (carmustine, etoposide, cytarabine, and melphalan) and autologous hematopoietic stem cell transplantation in December 1998. The disease recurred at the same location in November 1999. The patient was treated with 4 cycles of standard bleomycin, etoposide, doxorubicin, cyclophosphamide, vincristine, procarbazine, and prednisone therapy, with complete remission. However, this discrete lesion reappeared in February 2001. Because this localized HD was not sensitive to chemotherapy and complementary irradiation was excluded be-

From the Clinic of Hematology, ${ }^{a}$ Department of Thoracic Surgery, ${ }^{\mathrm{b}}$ and the Department of Pathology, ${ }^{\mathrm{c}}$ ULB-Hôpital Erasme, Brussels, Belgium.

Received for publication Aug 3, 2004; accepted for publication Aug 23, 2004.

Address for reprints: A. Kentos, MD, Clinic of Hematology, ULB-Hôpital Erasme, 808, Route de Lennik, 1070 Bruxelles, Belgium (E-mail: akentos@ulb.ac.be).

J Thorac Cardiovasc Surg 2005;129:1172

$0022-5223 / \$ 30.00$

Copyright (C) 2005 by The American Association for Thoracic Surgery

doi:10.1016/j.jtcvs.2004.08.039 cause of location at the margin of the previously irradiated field, a surgical approach was elected. The patient underwent a left upper pulmonary lobectomy with radical mediastinal lymph node dissection in March 2001. The pathologic examination confirmed the presence of a discrete, well-defined lesion showing the histologic characteristics of HD of the nodular sclerosis subgroup, without lymph node dissemination. Complete remission persists as of May 2004, with negative results of fluorodeoxyglucose positron emission tomographic scan and no evidence of recurrence on chest computed tomography.

\section{Discussion}

Patients with advanced HD that relapses after first-line chemotherapy may have further remissions with salvage treatment. Highdose chemotherapy and transplantation of hematopoietic stem cells improves freedom from treatment failure in patients with chemosensitive first relapse of $\mathrm{HD}$, irrespective of delay after initial remission. ${ }^{1-3}$ Unfortunately, relapse or disease progression after autologous transplantation occurs in a significant proportion of patients with $\mathrm{HD}$, and no clear-cut therapeutic guidelines exist for these patients for whom both first-line and high-dose salvage regimens have failed. ${ }^{4}$ Gemcitabine, monoclonal antibodies, and active immunotherapy may be tested as alternatives to the option of conventional or nonmyeloablative allogeneic transplantation. ${ }^{3,5}$ The favorable course observed in our patient suggests that elective surgery might be an alternative for highly selected patients with recurrent localized $\mathrm{HD}$, especially pulmonary recurrence.

\section{References}

1. Schmitz N, Pfistner B, Sextro M, Sieber M, Carella AM, Haenel M, et al. Aggressive conventional chemotherapy compared with high-dose chemotherapy with autologous haemopoietic stem-cell transplantation for relapsed chemosensitive Hodgkin's disease: a randomised trial. Lancet. 2002;359:2065-71.

2. Diehl V, Stein H, Hummel M, Zollinger R, Connors JM. Hodgkin's lymphoma: biology and treatment strategies for primary, refractory, and relapsed disease. Hematology (Am Soc Hematol Educ Program). 2003: 225-47.

3. Diehl V, Thomas RK, Re D. Part II: Hodgkin's lymphoma-diagnosis and treatment. Lancet Oncol. 2004;5:19-26.

4. Paltiel O, Rubinstein C, Or R, Nagler A, Gordon L, Deutsch L, Polliack A, et al. Factors associated with survival in patients with progressive disease following autologous transplant for lymphoma. Bone Marrow Transplant. 2003;31:565-9.

5. Zinzani PL, Tani M, Gabriele A, Gherlinzoni F, de Vivo A, Ricci P, et al. High-dose therapy with autologous transplantation for Hodgkin's disease: the Bologna experience. Haematologica. 2003;88:522-8. 